\title{
THE BLOWING-UP PHENOMENON FOR A REACTION DIFFUSION EQUATION WITH A LOCALIZED NON LINEAR SOURCE TERM AND DIRICHLET-NEUMANN BOUNDARY CONDITIONS
}

\section{HALIMA NACHID ${ }^{1,2,3}$, FIRMIN K. N'GOHISSE ${ }^{4}$ and YORO GOZO ${ }^{1}$}

1Département de Mathématiques et Informatiques

Université Nangui Abrogoua, UFR-SFA

02 BP 801 Abidjan 02

Côte d'Ivoire

e-mail: yorocarol@yahoo.fr

2International University of Grand-Bassam

Route de Bonoua Grand-Bassam

BP 564 Grand-Bassam

Côte d'Ivoire

${ }^{3}$ Laboratoire de Modélisation Mathematique

et de Calcul Économique LM2CE Settat

Maroc

e-mail: halimanachid@yahoo.fr

2020 Mathematics Subject Classification: 35B40, 35B50, 35K60, 65M06.

Keywords and phrases: discretization, numerical blow-up time, blow-up, Dirichlet, Neumann boundary conditions, convergence, localized nonlinear source term.

Received October 10, 2019; Revised November 20, 2019

(C) 2020 Scientific Advances Publishers

This work is licensed under the Creative Commons Attribution International License (CC BY 3.0).

http://creativecommons.org/licenses/by/3.0/deed.en_US

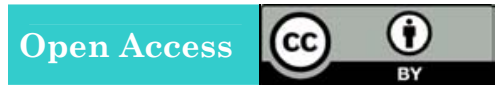


${ }^{4}$ Département de Mathématiques et Informatiques

Université Peleforo Gon Coulibaly de Korhogo

UFR-Sciences Biologiques

BP 1328 Korhogo

Côte d'Ivoire

e-mail: firmingoh@yahoo.fr

\begin{abstract}
In this paper, we study a localized nonlinear reaction diffusion equation. We investigate interactions among the localized and local sources, nonlinear diffusion with the zero boundary value condition to establish the blow-up solution and estimate the numerical approximation for the following initialboundary value problem:

$$
\left\{\begin{array}{l}
u_{t}(x, t)=u_{x x}(x, t)+f\left(u\left(x_{0}, t\right)\right), \quad(x, t) \in(-1,1) \times(0, T) \\
u(-1, t)=0, \quad u_{x}(1, t)=0, \quad t \in(0, T) \\
u(x, 0)=u_{0}(x) \geq 0, \quad x \in[-1,1]
\end{array}\right.
$$

where $f(s)$ is a positive, increasing, convex function for the nonnegative values of $s, \int_{0}^{+\infty} \frac{d s}{f(s)}<+\infty, x_{0}=1, u_{0} \in C^{1}([-1,1]), u_{0}(-1)=0, u_{0}^{\prime}(1)=0$.

We find some conditions under which the solution of a discrete form of the above problem blows up in a finite time and a numerical method is proposed for estimating its numerical blow-up time. We also prove the convergence of the numerical blow-up time to the theoretical one. Finally, we give some numerical results to illustrate our analysis.
\end{abstract}

\title{
1. Introduction
}

Consider the following initial-boundary value problem:

$$
\begin{gathered}
u_{t}(x, t)=u_{x x}(x, t)+f\left(u\left(x_{0}, t\right)\right), \quad(x, t) \in(-1,1) \times(0, T), \\
u(-1, t)=0, \quad u_{x}(1, t)=0, \quad t \in(0, T), \\
u(x, 0)=u_{0}(x) \geq 0, \quad x \in[-1,1]
\end{gathered}
$$

where $f(s)$ is a positive, increasing, convex function for the nonnegative values of $s, \int_{0}^{+\infty} \frac{d s}{f(s)}<+\infty, x_{0}=1, u_{0} \in C^{1}([-1,1]), u_{0}(-1)=0, u_{0}^{\prime}(1)=0$, 
which models the temperature distribution of a large number of physical phenomena from physics, chemistry and biology. The particularity of the problem described in (1.1)-(1.3) is that it represents a model in physical phenomena where the reaction is driven by the temperature at a single site. This kind of phenomena is observed in biological and chemical diffusion processes in which the reaction takes place only at some local sites. This model is appropriate to describe:

(i) The influence of defect structures on a catalytic surface.

(ii) The temperature in a solid-fuel combustion scenario where the heat that is input into the system is localized, say as in a laser focused on one spot in the domain.

(iii) Chemical reaction-diffusion processes in which, due to effect of catalyst, the reaction takes place only at a single site.

(iv) A heat stationary source which can support an explosive reaction. A stationary source provides a continuous supply of heat to the same environment.

(v) The ignition of a combustible medium with damping, where either a heated wire or a pair of small electrodes supplies a large amount of energy to every confined area.

For more physical motivation, see [8].

$\left(A_{0}\right) u_{0}:[-1,1] \rightarrow[0, \infty)$ is a positive, nondecreasing $c^{1}$ function. $u_{0}(-1)=0, u_{0}^{\prime}(1)=0$,

$\left(A_{f}\right) f:[0, \infty) \rightarrow[0, \infty)$ is a positive, increasing, convex function for the nonnegative values of $s, c^{1}$ function, $\int_{0}^{+\infty} \frac{d s}{f(s)}<+\infty$. Here $(0, T)$ is the maximal time interval of existence of the solution $u$. The time $T$ may be finite or infinite. When $T$ is infinite, we say that the solution $u$ exists globally. When $T$ is finite, the solution $u$ develops a singularity in a finite time, namely,

$$
\lim _{t \rightarrow T}\|u(., t)\|_{\infty}=+\infty,
$$

where $\|u(., t)\|_{\infty}=\max _{-1 \leq x \leq 1}|u(x, t)|$. 
In this case, we say that the solution $u$ blows up in a finite time and the time $T$ is called the blow-up time of the solution $u$.

This kind of the phenomena where the solutions of localized nonlinear heat equations which blow up in a finite time have been the subject of investigation of many authors (see [17], [44]-[46] and the references cited therein). In particular, the above problem has been studied and existence and uniqueness of a classical solution has been proved. Under some assumptions, it is also shown that the classical solution blows up in a finite time (see [17], [44], [45]).

In this paper, we are interesting in the numerical study of the above problem. Firstly, we show that under some assumptions, the solution of a discrete form of (1.1)-(1.3) blows up in a finite time and estimate its numerical blow-up time. We also show that the numerical blow-up time converges to the real one when the mesh size goes to zero. At the end of the paper, we have shown how one may treat the case of Dirichlet boundary conditions. One may find in [1]-[7], [12], [13], [16], [18]-[28], [31]-[33], [41] similar studies concerning other parabolic problems. Let us notice that many authors have used numerical methods to study the phenomenon of blow-up but there are only a few studies on the convergence of the numerical blow-up time for solutions which blow up in $L^{\infty}$ norm. For instance in [2], the authors have proved the convergence of numerical blow-up time for solutions which blow up in $L^{p}$ norm with $1<p<\infty$.

The rest of the paper is organized as follows. In the next section, we give some results which will be used later. In the third section, under some assumptions, we show that the solution of a discrete form of (1.1)-(1.3) blows up in a finite time and estimate its numerical blow-up time. In the fourth section, we show that, under some additional hypotheses, the numerical blow-up time goes to the real one when the mesh size goes to zero. Finally, in the last section, we give some numerical results to illustrate our analysis. 


\section{The Discrete Blow-up Solution}

In this section, we study the phenomenon of blow-up using a full discrete explicit scheme of (1)-(3). We start by the construction of a scheme as follows. Let $I$ be a positive integer, where $h=\frac{1}{I}$ is the mesh parameter and define the grid $x_{i}=i h$ or $x_{i+1}=x_{i}+h$ and $\Delta t_{n}=x_{i+1}-x_{i}$ the step size, $\varphi_{i}$ is the discrete approximation of the initial data.

$\left(A_{\varphi_{i}}\right) \varphi_{i}:[0, I] \rightarrow[0, \infty)$ is a positive function. Approximate the problem (1.1)-(1.3) by the solution $U_{h}^{(n)}=\left(U_{0}^{(n)}, U_{1}^{(n)}, \ldots, U_{I}^{(n)}\right)^{T}$, and approximate the initial condition $u_{0}(x)$ by $U_{i}^{(0)}=\varphi_{i}$ of the following discrete equations:

$$
\begin{gathered}
\delta_{t} U_{i}^{(n)}=\delta^{2} U_{i}^{(n)}+f\left(U_{I}^{(n)}\right), \quad 1 \leq i \leq I, \\
U_{0}^{(n)}=0, \\
U_{i}^{(0)}=\varphi_{i} \geq 0, \quad 1 \leq i \leq I,
\end{gathered}
$$

where $n \geq 0, \varphi_{i+1} \geq \varphi_{i}, 0 \leq i \leq I-1$,

$$
\begin{gathered}
\delta^{2} U_{i}^{(n)}=\left\{\begin{array}{l}
\frac{U_{i+1}^{(n)}-2 U_{i}^{n}+U_{i-1}^{(n)}}{h^{2}}, \quad 1 \leq i \leq I-1, \\
\frac{2}{h^{2}}\left(U_{I-1}^{(n)}-U_{I}^{(n)}\right), \quad i=I
\end{array}\right. \\
\delta_{t} U_{i}^{(n)}=\frac{U_{i}^{(n+1)}-U_{i}^{(n)}}{\Delta t_{n}}
\end{gathered}
$$


then

$$
\begin{gathered}
\frac{U_{i}^{(n+1)}-U_{i}^{(n)}}{\Delta t_{n}}-\frac{U_{i+1}^{(n)}-2 U_{i}^{n}+U_{i-1}^{(n)}}{h^{2}}=f\left(U_{i}^{(n)}\right), \quad 1 \leq i \leq I-1, \\
U_{0}^{(n)}=0 \\
U_{i}^{(0)}=\varphi_{i} \geq 0, \quad 1 \leq i \leq I, \\
\frac{2}{h^{2}}\left(U_{I-1}^{(n)}-U_{I}^{(n)}\right)+\frac{U_{I}^{(n+1)}-U_{I}^{(n)}}{\Delta t_{n}}=f\left(U_{I}^{(n)}\right), \quad i=I,
\end{gathered}
$$

where

$$
\Delta t_{n}=\min \left\{\frac{h^{2}}{3}, \frac{\tau}{f\left(\left\|U_{h}^{(n)}\right\|_{\infty}\right)}\right\}, \quad 0<\tau<1 .
$$

Let us notice that the restriction on the time step ensure the positivity of the discrete solution.

Definition 2.1. We say that the solution $u$ of (1.1)-(1.3) blows up in a finite time, if there exist a finite time $T$ such that $\|u(\cdot, t)\|_{\infty}<\infty$ for $t \in[0, T)$ but

$$
\lim _{t \rightarrow T}\|u(\cdot, t)\|_{\infty}=\infty,
$$

where $\|u(\cdot, t)\|_{\infty}=\sup _{x \in \Omega}|u(x, t)|$ and the time $T$ is called the blow-up time of the solution $u$, when $T$ is infinite, we say that the solution $u$ exists globally.

\section{Properties of the Discrete Scheme}

In this section, we give some important results which will be used later. The following Lemmas 3.1 to 3.4 are a form of the maximum principal for discrete equations. 
Lemma 3.1. Let $U_{h}^{(n)}$ be the solution of (2.1)-(2.3). Then we have $U_{i+1}^{(n)} \geq U_{i}^{(n)}, 0 \leq i \leq I-1$.

Proof. Let $Z_{i}^{(n)}=U_{i+1}^{(n)}-U_{i}^{(n)}, 0 \leq i \leq I-1$. Obviously $Z_{0}^{(n)} \geq 0$ because $\varphi_{i+1} \geq \varphi_{i}$.

A routine computation reveals that $Z_{i}^{(n+1)}=U_{i+1}^{(n+1)}-U_{i}^{(n+1)}$ then

$$
\begin{gathered}
\frac{Z_{i}^{(n+1)}-Z_{i}^{(n)}}{\Delta t_{n}}=\frac{Z_{i+1}^{(n)}-2 Z_{i}^{(n)}+Z_{i-1}^{(n)}}{h^{2}}, 1 \leq i \leq I-2, \\
\frac{Z_{I-1}^{(n+1)}-Z_{I-1}^{(n)}}{\Delta t_{n}}=\frac{-3 Z_{I-1}^{(n)}+Z_{I-2}^{(n)}}{h^{2}}, i=I-1,
\end{gathered}
$$

which implies that

$$
\begin{gathered}
Z_{i}^{(n+1)}=\frac{\Delta t_{n}}{h^{2}} Z_{i+1}^{(n)}+\left(1-\frac{2 \Delta t_{n}}{h^{2}}\right) Z_{i}^{(n)}+\frac{\Delta t_{n}}{h^{2}} Z_{i-1}^{(n)}, \quad 1 \leq i \leq I-2, \\
Z_{I-1}^{(n+1)}=\frac{\Delta t_{n}}{h^{2}} Z_{I-2}^{(n)}+\left(1-\frac{3 \Delta t_{n}}{h^{2}}\right) Z_{I-1}^{(n)} .
\end{gathered}
$$

Since $Z_{i}^{(0)} \geq 0,1 \leq i \leq I-1$, we deduce by induction that $Z_{i}^{(n)} \geq 0$, $0 \leq i \leq I-1$ and the proof is complete.

Lemma 3.2. Let $a^{(n)}$ be a nonnegative sequence and let $V_{h}^{(n)}$ be a sequence such that

$$
\begin{gathered}
\delta_{t} V_{i}^{(n)}-\delta^{2} V_{i}^{(n)}-a^{(n)} V_{i}^{(n)} \geq 0, \quad 1 \leq i \leq I, \quad n \geq 0, \\
V_{0}^{(n)} \geq 0, \quad n \geq 0 \\
V_{i}^{(0)} \geq 0, \quad 0 \leq i \leq I .
\end{gathered}
$$

Then $V_{i}^{(n)} \geq 0$ for $n \geq 0,0 \leq i \leq I$, if $\Delta t_{n} \leq \frac{h^{2}}{3}$. 
Proof. A routine calculation gives

$$
\frac{V_{i}^{(n+1)}-V_{i}^{(n)}}{\Delta t_{n}}-\frac{V_{i+1}^{(n)}-2 V_{i}^{(n)}+V_{i-1}^{(n)}}{h^{2}}-a^{(n)} V_{i}^{(n)} \geq 0, \quad 1 \leq i \leq I-1,
$$

which implies that

$$
\begin{gathered}
V_{i}^{(n+1)} \geq \frac{\Delta t_{n}}{h^{2}} V_{i+1}^{(n)}+\left(1-2 \frac{\Delta t_{n}}{h^{2}}\right) V_{i}^{(n)}+\frac{\Delta t_{n}}{h^{2}} V_{i-1}^{(n)}+\Delta t_{n} a^{(n)} V_{I}^{(n)}, 1 \leq i \leq I-1, \\
V_{I}^{(n+1)} \geq \frac{2 \Delta t_{n}}{h^{2}} V_{I-1}^{(n)}+\left(1-2 \frac{\Delta t_{n}}{h^{2}}\right) V_{I}^{(n)}+\Delta t_{n} a^{(n)} V_{I}^{(n)}
\end{gathered}
$$

Since $\Delta t_{n} \leq \frac{h^{2}}{3}$, we see that $1-2 \frac{\Delta t_{n}}{h^{2}}$ is nonnegative. Due to the fact that $V_{h}^{(0)} \geq 0$, we deduce by induction that $V_{h}^{(n)} \geq 0$ for $n \geq 0$, which ends the proof.

A direct consequence of the above result is the following comparison lemma. Its proof is straightforward.

Lemma 3.3. Suppose that $a^{(n)}$ and $b^{(n)}$ are two sequences such that $a^{(n)}$ is nonnegative. Let $V_{h}^{(n)}$ and $W_{h}^{(n)}$ two sequences such that

$$
\begin{gathered}
\delta_{t} V_{i}^{(n)}-\delta^{2} V_{i}^{(n)}-a^{(n)} V_{i}^{(n)}+b^{(n)} \leq \delta_{t} W_{i}^{(n)}-\delta^{2} W_{i}^{(n)}-a^{(n)} W_{i}^{(n)}+b^{(n)}, \\
1 \leq i \leq I, n \geq 0, \\
V_{0}^{(n)} \leq W_{0}^{(n)}, \quad n \geq 0, \\
V_{i}^{(0)} \leq W_{i}^{(0)}, \quad 0 \leq i \leq I .
\end{gathered}
$$

Then $V_{i}^{(n)} \leq W_{i}^{(n)}$ for $n \geq 0,0 \leq i \leq I$, if $\Delta t_{n} \leq \frac{h^{2}}{3}$.

Now, let us give the property of operator $\delta_{t}$. 
Lemma 3.4. Let $f:[0, \infty) \rightarrow[0, \infty)$ be a $C^{1}$ convex function and $U_{h}^{(n)} \in \mathbb{R}$ be a sequence such that $U_{h}^{(n)} \geq 0$. Then we have

$$
\begin{gathered}
\delta_{t} f\left(U_{h}^{(n)}\right) \geq f^{\prime}\left(U_{h}^{(n)}\right) \delta_{t} U_{h}^{(n)}, \quad n \geq 0, \\
\delta^{2} f\left(U_{h}^{(n)}\right) \geq f^{\prime}\left(U_{h}^{(n)}\right) \delta^{2} U_{h}^{(n)} .
\end{gathered}
$$

Proof. We apply Taylor's expansion to obtain

$$
f\left(U_{h}^{(n+1)}\right)=f\left(U_{h}^{(n)}\right)+\left(U_{h}^{(n+1)}-\left(U_{h}^{(n)}\right)\right) f^{\prime}\left(U_{h}^{(n)}\right)+\frac{\left(U_{h}^{(n+1)}-\left(U_{h}^{(n)}\right)\right)^{2}}{2} f^{\prime \prime}(\theta),
$$

where $\theta$ is an intermediate value between $U_{h}^{(n+1)}$ and $U_{h}^{(n)}$;

$f\left(U_{h}^{(n-1)}\right)=f\left(U_{h}^{(n)}\right)+\left(U_{h}^{(n-1)}-\left(U_{h}^{(n)}\right)\right) f^{\prime}\left(U_{h}^{(n)}\right)+\frac{\left(U_{h}^{(n-1)}-\left(U_{h}^{(n)}\right)\right)^{2}}{2} f^{\prime \prime}(\tau)$,

where $\tau$ is an intermediate value between $U_{h}^{(n-1)}$ and $U_{h}^{(n)}$.

These two equations above imply that

$$
\begin{gathered}
f\left(U_{h}^{(n+1)}\right)-2 f\left(U_{h}^{(n)}\right)+f\left(U_{h}^{(n-1)}\right)=\left(U_{h}^{(n+1)}-2 U_{h}^{(n)}+U_{h}^{(n-1)}\right) f^{\prime}\left(U_{h}^{(n)}\right) \\
+\frac{\left(U_{h}^{(n+1)}-U_{h}^{(n)}\right)^{2}}{2} f^{\prime \prime}(\theta)+\frac{\left(U_{h}^{(n-1)}-U_{h}^{(n)}\right)^{2}}{2} f^{\prime \prime}(\tau) \\
\frac{f\left(U_{h}^{(n+1)}\right)-2 f\left(U_{h}^{(n)}\right)+f\left(U_{h}^{(n-1)}\right)}{h^{2}}=\frac{\left(U_{h}^{(n+1)}-2 U_{h}^{(n)}+U_{h}^{(n-1)}\right)}{h^{2}} f^{\prime}\left(U_{h}^{(n)}\right) \\
+\frac{\left(U_{h}^{(n+1)}-U_{h}^{(n)}\right)^{2}}{2 h^{2}} f^{\prime \prime}(\theta)+\frac{\left(U_{h}^{(n-1)}-U_{h}^{(n)}\right)^{2}}{2 h^{2}} f^{\prime \prime}(\tau) .
\end{gathered}
$$

The first equation and the last one imply that

$$
\delta^{2} f\left(U_{h}^{(n)}\right)=f^{\prime}\left(U_{h}^{(n)}\right) \delta^{2} U_{h}^{(n)}+\frac{\left(U_{h}^{(n+1)}-U_{h}^{(n)}\right)^{2}}{2 h^{2}} f^{\prime \prime}(\theta)+\frac{\left(U_{h}^{(n-1)}-U_{h}^{(n)}\right)^{2}}{2 h^{2}} f^{\prime \prime}(\tau),
$$


and

$$
\delta_{t} f\left(U_{h}^{(n)}\right)=f^{\prime}\left(U_{h}^{(n)}\right) \delta_{t} U_{h}^{(n)}+\frac{1}{2} \Delta t_{n} \delta_{t}\left(U_{h}^{(n)}\right)^{2} f^{\prime \prime}(\theta) .
$$

Use the fact that $U_{h}^{(n)} \geq 0$ for $n \geq 0$ and using the convexcity of $f$ we obtain the desire result.

\section{Blow-up Time in the Discrete Solution}

In this section, under some assumptions, we show that the discrete solution blows up in a finite time and its numerical blow-up time converges to the real one when the mesh size tends to zero.

Definition 4.1. We say that the solution $U_{h}^{(n)}$ of (2.1)-(2.3) blows up in a finite time, if $\left\|U_{h}^{(n)}\right\|_{\infty}=+\infty$, and $T_{h}^{\Delta t}=\lim _{n \rightarrow \infty} \sum_{i=0}^{n-1} \Delta t_{i}<+\infty$, the number $T_{h}^{\Delta t}$ is called the numerical blow-up time of the solution $U_{h}^{(n)}$.

The following theorem shows that the discrete solution blows up under some conditions.

Theorem 4.1. Suppose that there exists a positive constant $A \in(0,1]$ such that the initial data at (2.3) satisfies:

$$
\begin{gathered}
\delta^{2} \varphi_{i}+f\left(\varphi_{i}\right) \geq 0,0 \leq i \leq I-1, \delta^{2} \varphi_{I}+f\left(\varphi_{I}\right) \geq A f\left(\varphi_{I}\right), \\
\delta^{2} \varphi_{I}+f\left(\varphi_{I}\right) \geq A f\left(\varphi_{I}\right),
\end{gathered}
$$

with $\varphi_{i}$ is an approximation of the initial data. Then the solution $U_{h}^{(n)}$ of (2.1)-(2.3) blows up in a finite time and its numerical blow-up time $T_{h}^{\Delta t}$ is estimated as follows:

$$
T_{h}^{\Delta t} \leq \frac{\tau}{f\left(\left\|\varphi_{h}\right\|_{\infty}\right)}+\frac{\tau}{\tau^{\prime}} \int_{\left\|\varphi_{h}\right\|_{\infty}}^{+\infty} \frac{d s}{f(s)}
$$

where $\tau^{\prime}=\min \left\{\frac{h^{2}}{3} f\left(\left\|\varphi_{h}\right\|_{\infty}\right), \tau\right\}$. 
Proof. Introduce the vector $J_{h}^{(n)}$ such that

$$
J_{i}^{(n)}=\delta_{t} U_{i}^{(n)}, 0 \leq i \leq I-1, J_{I}^{(n)}=\delta_{t} U_{I}^{(n)}-A f\left(U_{I}^{(n)}\right)
$$

A straightforward computation yields

$$
\begin{gathered}
\delta_{t} J_{i}^{(n)}-\delta^{2} J_{i}^{(n)}=\delta_{t}\left(\delta_{t} U_{i}^{(n)}-\delta^{2} U_{i}^{(n)}\right), 0 \leq i \leq I-1, n \geq 0, \\
\delta_{t} J_{I}^{(n)}-\delta^{2} J_{I}^{(n)}=\delta_{t}\left(\delta_{t} U_{I}^{(n)}-\delta^{2} U_{I}^{(n)}\right)-A \delta_{t} f\left(U_{I}^{(n)}\right)+A \delta^{2} f\left(U_{I}^{(n)}\right) .
\end{gathered}
$$

Using (2.4), we arrive at

$$
\begin{gathered}
\delta_{t} J_{i}^{(n)}-\delta^{2} J_{i}^{(n)}=\delta_{t} f\left(U_{i}^{(n)}\right), \quad 1 \leq i \leq I-1, \\
\delta_{t} J_{I}^{(n)}-\delta^{2} J_{I}^{(n)}=(1-A) \delta_{t} f\left(U_{I}^{(n)}\right)+A \delta^{2} f\left(U_{I}^{(n)}\right) .
\end{gathered}
$$

It follows from Lemma 3.4 that

$$
\begin{gathered}
\delta_{t} J_{i}^{(n)}-\delta^{2} J_{i}^{(n)} \geq f^{\prime}\left(U_{i}^{(n)}\right) \delta_{t} U_{i}^{(n)}, \quad 1 \leq i \leq I-1, \\
\delta_{t} J_{I}^{(n)}-\delta^{2} J_{I}^{(n)} \geq(1-A) f^{\prime}\left(U_{I}^{(n)}\right) \delta_{t} U_{I}^{(n)}+A f^{\prime}\left(U_{I}^{(n)}\right) \delta^{2} U_{I}^{(n)} .
\end{gathered}
$$

Taking into account (2.4), we deduce that

$$
\begin{gathered}
\delta_{t} J_{i}^{(n)}-\delta^{2} J_{i}^{(n)} \geq f^{\prime}\left(U_{i}^{(n)}\right) \delta_{t} U_{i}^{(n)}-A f^{\prime}\left(U_{i}^{(n)}\right) f\left(U_{i}^{(n)}\right), \\
\delta_{t} J_{I}^{(n)}-\delta^{2} J_{I}^{(n)} \geq f^{\prime}\left(U_{I}^{(n)}\right) \delta_{t} U_{I}^{(n)}-A f^{\prime}\left(U_{I}^{(n)}\right) f\left(U_{I}^{(n)}\right),
\end{gathered}
$$

which implies that

$$
\delta_{t} J_{i}^{(n)}-\delta^{2} J_{i}^{(n)} \geq f^{\prime}\left(U_{i}^{(n)}\right) J_{i}^{(n)}, \quad 1 \leq i \leq I .
$$

Obviously, we have $J_{0}^{(n)}=0$. From (4.1), we obtain $J_{i}^{(0)} \geq 0$. It follows from Lemma 3.2 that $J_{i}^{(n)} \geq 0,0 \leq i \leq I$. Hence, we have

$$
\frac{U_{I}^{(n+1)}-U_{I}^{(n)}}{\Delta t_{n}} \geq A f\left(U_{I}^{(n)}\right) .
$$


Consequently, we get

$$
U_{I}^{(n+1)} \geq U_{I}^{(n)}+A \Delta t_{n} f\left(U_{I}^{(n)}\right) .
$$

Since $U_{I}^{(n)}=\left\|U_{h}^{(n)}\right\|_{\infty}$, we arrive at

$$
\left\|U_{h}^{(n+1)}\right\|_{\infty} \geq\left\|U_{h}^{(n)}\right\|_{\infty}+A \Delta t_{n} f\left(\left\|U_{h}^{(n)}\right\|_{\infty}\right) .
$$

It not difficult to see that

$$
\Delta t_{n} f\left(\left\|U_{h}^{(n)}\right\|_{\infty}\right)=\min \left\{\frac{h^{2}}{3} f\left(\left\|U_{h}^{(n)}\right\|_{\infty}\right), \tau\right\} .
$$

From (4.7), we get $\left\|U_{h}^{(n+1)}\right\|_{\infty} \geq\left\|U_{h}^{(n)}\right\|_{\infty}$ and by induction we obtain $\left\|U_{h}^{(n)}\right\|_{\infty} \geq\left\|U_{h}^{(0)}\right\|_{\infty}=\left\|\varphi_{h}\right\|_{\infty}$. It follows that

$$
\Delta t_{n} f\left(\left\|U_{h}^{(n)}\right\|_{\infty}\right) \geq \min \left\{\frac{h^{2}}{3} f\left(\left\|\varphi_{h}\right\|_{\infty}\right), \tau\right\}=\tau^{\prime}
$$

Consequently, we have

$$
\left\|U_{h}^{(n+1)}\right\|_{\infty} \geq\left\|U_{h}^{(n)}\right\|_{\infty}+\tau^{\prime}
$$

Using a recursion argument, we discover that

$$
\left\|U_{h}^{(n)}\right\|_{\infty} \geq\left\|U_{h}^{(0)}\right\|_{\infty}+n \tau^{\prime}=\left\|\varphi_{h}\right\|_{\infty}+\tau^{\prime} .
$$

Hence, we see that $\left\|U_{h}^{(n)}\right\|_{\infty}$ goes to infinity as $n$ approaches infinity. Now let us estimate the numerical blow-up time. From the restriction on the time step, we get

$$
\sum_{n=0}^{\infty} \Delta t_{n} \leq \sum_{n=0}^{\infty} \frac{\tau}{f\left(\left\|U_{h}^{(n)}\right\|_{\infty}\right)}
$$

Due to (4.8), we arrive at

$$
\sum_{n=0}^{\infty} \Delta t_{n} \leq \sum_{n=0}^{\infty} \frac{\tau}{f\left(\left\|\varphi_{h}\right\|_{\infty}+n \tau^{\prime}\right)}
$$


We observe that

$$
\begin{aligned}
\int_{0}^{+\infty} \frac{d s}{f\left(\left\|\varphi_{h}\right\|_{\infty}+s \tau^{\prime}\right)}=\sum_{n=0}^{\infty} \int_{n}^{n+1} & \frac{d s}{f\left(\left\|\varphi_{h}\right\|_{\infty}+s \tau^{\prime}\right)} \\
& \geq \sum_{n=0}^{\infty} \frac{1}{f\left(\left\|\varphi_{h}\right\|_{\infty}+(n+1) \tau^{\prime}\right)}
\end{aligned}
$$

Since

$$
\int_{0}^{+\infty} \frac{d s}{f\left(\left\|\varphi_{h}\right\|_{\infty}+s \tau^{\prime}\right)}=\frac{1}{\tau^{\prime}} \int_{\left\|\varphi_{h}\right\|_{\infty}}^{+\infty} \frac{d s}{f(s)}
$$

we deduce that

$$
\sum_{n=0}^{\infty} \Delta t_{n} \leq \frac{\tau}{f\left(\left\|\varphi_{h}\right\|_{\infty}\right)}+\frac{\tau}{\tau^{\prime}} \int_{\left\|\varphi_{h}\right\|_{\infty}}^{+\infty} \frac{d s}{f(s)}
$$

Use the fact that the quantity on the right hand side of the above inequality is finite to complete the rest of the proof.

Remark 4.1. From (4.8),

$$
\left\|U_{h}^{(n+1)}\right\|_{\infty} \geq\left\|U_{h}^{(n)}\right\|_{\infty}+\tau^{\prime},
$$

we get by induction that

$$
\left\|U_{h}^{(n)}\right\|_{\infty} \geq\left\|U_{h}^{(q)}\right\|_{\infty}+\tau^{\prime}(n-q)
$$

Hence

$$
T_{h}^{\Delta t}-T_{q}=\sum_{n=q}^{\infty} \Delta t_{n} \leq \sum_{n=q}^{\infty} \frac{\tau}{f\left(\left\|U_{h}^{(n)}\right\|_{\infty}\right)} \leq \sum_{n=q}^{\infty} \frac{\tau}{f\left(\left\|U_{h}^{(q)}\right\|_{\infty}+(n-q) \tau^{\prime}\right)}
$$

where $t_{q}=\sum_{n=q}^{\infty} \Delta t_{n}$. We observe that

$$
\begin{aligned}
\int_{0}^{+\infty} \frac{d s}{f\left(\left\|U_{h}^{(q)}\right\|_{\infty}+s \tau^{\prime}\right)} & =\sum_{n=0}^{\infty} \int_{n}^{n+1} \frac{d s}{f\left(\left\|U_{h}^{(q)}\right\|_{\infty}+s \tau^{\prime}\right)} \\
& \geq \sum_{n=0}^{\infty} \frac{1}{f\left(\left\|U_{h}^{(q)}\right\|_{\infty}+(n+1) \tau^{\prime}\right)} .
\end{aligned}
$$


Since

$$
\int_{0}^{+\infty} \frac{d s}{f\left(\left\|U_{h}^{(q)}\right\|_{\infty}+s \tau^{\prime}\right)}=\frac{1}{\tau^{\prime}} \int_{\left\|U_{h}^{(q)}\right\|_{\infty}}^{+\infty} \frac{d s}{f(s)},
$$

we get

$$
T_{h}^{\Delta t}-t_{q} \leq \frac{\tau}{f\left(\left\|U_{h}^{(q)}\right\|_{\infty}\right)}+\frac{\tau}{\tau^{\prime}} \int_{\left\|U_{h}^{(q)}\right\|_{\infty}}^{+\infty} \frac{d s}{f(s)}
$$

Since $\tau^{\prime}=\min \left\{\frac{h^{2}}{3} f\left(\left\|\varphi_{h}\right\|_{\infty}\right), \tau\right\}$, if we take $\tau=h^{2}$, we get

$$
\frac{\tau}{\tau^{\prime}}=\min \left\{\frac{1}{3} f\left(\left\|\varphi_{h}\right\|_{\infty}\right), 1\right\}
$$

which implies that there exists a positive constant $B$ such that $\frac{\tau}{\tau^{\prime}} \leq B$.

\section{Convergence of the Blow-up Time}

In this section, under some conditions, we show that the discrete solution blows up in a finite time and that its numerical blow-up time goes to the analytic one when the mesh size goes to zero.

In order to prove the convergence of the discrete blow-up time, we need to show that the discrete scheme converges for each fixed time interval $[0, T]$. We denote by $u_{h}\left(t_{n}\right)=\left(u\left(x_{0}, t_{n}\right), \ldots, u\left(x_{I}, t_{n}\right)\right)^{T}$ and state the result on the convergence of our scheme by the following.

Theorem 5.1. Suppose that the problem (1.1)-(1.3) has a solution $u \in C^{4,2}([-1,1] \times[0, T])$, and that $U_{h}^{(n)}$ approximates the solution $u$ of (1.1)-(1.3) with $U_{h}^{(0)}=\varphi_{h}$. Assume that the initial data at (2.3) verifies

$$
\left\|\varphi_{h}-u_{h}(0)\right\|_{\infty}=o(1) \quad \text { as } \quad h \rightarrow 0 .
$$


Then the problem (2.1)-(2.3) has a solution $U_{h}^{(n)}$ for $h$ sufficiently small, $0 \leq n \leq J$ and we have the following estimate:

$$
\max _{0 \leq n \leq J}\left\|U_{h}^{(n)}-u_{h}\left(t_{n}\right)\right\|_{\infty}=O\left(\left\|\varphi_{h}-u_{h}(0)\right\|_{\infty}+h^{2}+\Delta t_{n}\right) \quad \text { as } \quad h \rightarrow 0,
$$

where $J$ is such that $\sum_{n=0}^{J-1} \Delta t_{n} \leq T$ and $t_{n}=\sum_{j=0}^{n-1} \Delta t_{j}$.

Proof. For each $h$, the problem (2.1)-(2.3) has a solution $U_{h}^{(n)}$, we want to proof that $U_{h}^{(n)}$ approaches to $u_{h}$ as $h \rightarrow 0$.

Let $N \leq J$ be the greatest value of $n$ such that

$$
\left\|U_{h}^{(n)}-u_{h}\left(t_{n}\right)\right\|_{\infty}<1 \quad \text { for } \quad n<N .
$$

We know that $N \geq 1$ because of (5.1). Due to the fact that $u \in C^{4,2}([-1,1] \times[0, T])$, there exists a positive constant $K$ such that $\|u\| \leq K$.

Applying the triangle inequality, we obtain

$$
\left\|U_{h}^{(n)}\right\|_{\infty} \leq\left\|u_{h}\left(t_{n}\right)\right\|_{\infty}+\left\|U_{h}^{(n)}-u_{h}\left(t_{n}\right)\right\|_{\infty} \leq 1+K .
$$

Since $u \in C^{4,2}([-1,1] \times[0, T])$, taking the derivative in $x$ on both sides of (1.1) and due to the fact that $u_{x}, u_{x t}$ vanish at $x=1$. We observe that $u_{x x x}$ also vanishes at $x=1$. Using Taylor's expansion, we find that

$$
\begin{array}{r}
\delta_{t} u\left(x_{i}, t_{n}\right)-\delta^{2} u\left(x_{i}, t_{n}\right)-f\left(u\left(x_{I}, t_{n}\right)\right)=-\frac{h^{2}}{12} u_{x x x x}\left(\tilde{x}_{i}, t_{n}\right)+\frac{\Delta t_{n}}{2} u_{t t}\left(x_{i}, \tilde{t}_{n}\right), \\
\text { for } 1 \leq i \leq I .
\end{array}
$$

To establish the above equality for $i=I$, we have used the fact that $u_{x x x}$ vanishes at $x=1$. Let $e_{h}^{(n)}=U_{h}^{(n)}-u_{h}\left(t_{n}\right)$ be the error of discretization. From the mean value theorem, we get 


$$
\delta_{t} e_{i}^{(n)}-\delta^{2} e_{i}^{(n)}=f^{\prime}\left(\varsigma_{i}^{(n)}\right) e_{i}^{(n)}+\frac{h^{2}}{12} u_{x x x x}\left(\tilde{x}_{i}, t_{n}\right)-\frac{\Delta t_{n}}{2} u_{t t}\left(x_{i}, \tilde{t}_{n}\right), 1 \leq i \leq I,
$$

where $\varsigma_{i}^{(n)}$ is an intermediate value between $u\left(x_{i}, t_{n}\right)$ and $U_{i}^{(n)}$. Since $u_{x x x x}(x, t), u_{t t}(x, t)$ are bounded, there exists a positive constant $M$ such that

$$
\delta_{t} e_{i}^{(n)}-\delta^{2} e_{i}^{(n)} \leq f^{\prime}\left(\varsigma_{i}^{(n)}\right) e_{i}^{(n)}+M \Delta t_{n}+M h^{2}, \quad 0 \leq i \leq I .
$$

Let $P=1+K$ and introduce the vector $V_{h}^{(n)}$ defined as follows:

$$
V_{i}^{(n)}=e^{(P+1) t_{n}}\left(\left\|\varphi_{h}-u_{h}(0)\right\|_{\infty}+M h^{2}+M \Delta t_{n}\right), \quad 0 \leq i \leq I
$$

A straightforward computation gives

$$
\begin{gathered}
\delta_{t} V_{i}^{(n)}-\delta^{2} V_{i}^{(n)} \geq f^{\prime}\left(\varsigma_{i}^{(n)}\right) V_{i}^{(n)}+M \Delta t_{n}+M h^{2}, \quad 1 \leq i \leq I, \\
V_{0}^{(n)} \geq e_{0}^{(n)}, \\
V_{i}^{(0)} \geq e_{i}^{(0)}, \quad 0 \leq i \leq I .
\end{gathered}
$$

We observe that $f^{\prime}\left(\varsigma_{i}^{(n)}\right)$ is bounded from above by $f(P)$. It follows from comparison Lemma 3.3 that $V_{h}^{(n)} \geq e_{h}^{(n)}$. By the same way, we also prove that $V_{h}^{(n)} \geq-e_{h}^{(n)}$ which implies that

$$
\left\|U_{h}^{(n)}-u_{h}\left(t_{n}\right)\right\|_{\infty} \leq e^{(P+1) t_{n}}\left(\left\|\varphi_{h}-u_{h}(0)\right\|_{\infty}+M h^{2}+M \Delta t_{n}\right) .
$$

Let us show that $N=J$.

Suppose that $N<J$, if we replace $n$ by $N$ in the above inequality and use (5.2), we find that

$$
1 \leq\left\|U_{h}^{(N)}-u_{h}\left(t_{N}\right)\right\|_{\infty} \leq e^{(P+1) t_{N}}\left(\left\|\varphi_{h}-u_{h}(0)\right\|_{\infty}+M h^{2}+M \Delta t_{n}\right) .
$$


Since term on the right hand side of the second inequality goes to zero as $h$ tends to zero, we deduce that $1 \leq 0$, which is a contradiction and the proof is complete.

Now, we are in a position to prove the main theorem of this section.

Theorem 5.2. Suppose that the problem (1.1)-(1.3) has a solution $u$ which blows up in a finite time $T_{0}$ and $u \in C^{4,2}\left([-1,1] \times\left[0, T_{0}\right)\right)$. Assume that the initial data at (2.3) satisfies

$$
\left\|\varphi_{h}-u_{h}(0)\right\|_{\infty}=o(1) \quad \text { as } \quad h \rightarrow 0 .
$$

Under the assumption of Theorem 4.1, the problem (2.1)-(2.3) has a solution $U_{h}^{(n)}$ which blows up in a finite time $T_{h}^{\Delta t}$ and the following relation holds:

$$
\lim _{h \rightarrow 0} T_{h}^{\Delta t}=T_{0}
$$

Proof. We know from Remark 4.1 that $\frac{\tau}{\tau^{\prime}}$ is bounded. Letting $\varepsilon>0$, there exists a constant $R>0$ such that

$$
\frac{\tau}{f(x)}+\frac{\tau}{\tau^{\prime}} \int_{x}^{+\infty} \frac{d s}{f(s)}<\frac{\varepsilon}{2} \quad \text { for } \quad x \in[R,+\infty)
$$

Since $u$ blows up at the time $T_{0}$, there exists $T_{1} \in\left(T_{0}-\frac{\varepsilon}{2}, T_{0}\right)$ such that

$$
\|u(., t)\|_{\infty} \geq 2 R \quad \text { for } \quad t \in\left[T_{1}, T_{0}\right) .
$$

Let $T_{2}=\frac{T_{1}+T_{2}}{2}$ and $q$ be a positive integer such that $t_{q}=\sum_{n=0}^{q-1} \Delta t_{n}$ $\in\left[T_{1}, T_{2}\right]$ for $h$ small enough. 
We have $\sup _{t \in\left[0, T_{2}\right]}\|u(., t)\|_{\infty}<+\infty$. It follows from Theorem 4.1 that the problem (2.1)-(2.3) has a solution $U_{h}^{(n)}$ which obeys

$$
\left\|U_{h}^{(n)}-u_{h}\left(t_{n}\right)\right\|_{\infty}<R \quad \text { for } \quad n \leq q
$$

which implies that

$$
\left\|U_{h}^{(q)}\right\|_{\infty} \geq\left\|u_{h}\left(t_{q}\right)\right\|_{\infty}-\left\|U_{h}^{(q)}-u_{h}\left(t_{q}\right)\right\|_{\infty} \geq R .
$$

From Theorem 4.1, $U_{h}^{(n)}$ blows up at the time $T_{h}^{\Delta t}$. It follows from Remark 4.1 and (5.6) that

$$
\left|T_{h}^{\Delta t}-t_{q}\right| \leq \frac{\tau}{f\left(\left\|U_{h}^{(q)}\right\|_{\infty}\right)}+\frac{\tau}{\tau^{\prime}} \int_{\left\|U_{h}^{(q)}\right\|_{\infty}}^{+\infty} \frac{d s}{f(s)} \leq \frac{\varepsilon}{2}
$$

because $\left\|U_{h}^{(q)}\right\|_{\infty} \geq R$. We deduce that

$$
\left|T_{0}-T_{h}^{\Delta t}\right| \leq\left|T_{0}-t_{q}\right|+\left|t_{q}-T_{h}^{\Delta t}\right| \leq \frac{\varepsilon}{2}+\frac{\varepsilon}{2} \leq \varepsilon
$$

and the proof is complete.

Remark 5.1. Consider the following initial-boundary value problem:

$$
\begin{gathered}
u_{t}(x, t)=u_{x x}(x, t)+f(u(0, t)), \quad(x, t) \in(-1,1) \times(0, T), \\
u(-1, t)=0, \quad u(1, t)=0, \quad t \in(0, T) \\
u(x, 0)=u_{0}(x) \geq 0, \quad x \in[-1,1]
\end{gathered}
$$

where $u_{0}(x)$ is a positive and symmetric function in $[-1,1]$ and $u_{0}^{\prime}(x) \geq 0$ for $x \in(-1,0)$. Since $u_{0}(x)$ is symmetric in $[-1,1]$, from the maximum principle $u$ is also symmetric in $[-1,1]$. We observe that $u x(0, t)=0$ because $u(x, t)=u(-x, t)$. 
Consider now the solution $v$ of the following initial-boundary value problem below:

$$
\begin{gathered}
z_{t}(x, t)=u_{x x}(x, t)+f(u(0, t)), \quad(x, t) \in(-1,0) \times(0, T), \\
z(-1, t)=0, \quad z_{x}(1, t)=0, \quad t \in(0, T) \\
z(x, 0)=u_{0}(x) \geq 0, \quad x \in[-1,0] .
\end{gathered}
$$

Since $u$ is symmetric, we have $\max _{-1 \leq x \leq 1}|u(x, t)|=\max _{-1 \leq x \leq 0}|u(x, t)|=$ $\max _{-1 \leq x \leq 0}|z(x, t)|$. Hence, to get an approximation of the blow-up time of the solution $u$, it suffices to obtain the one of the classical solution $z$ which has been the subject of investigation of the present paper.

\section{Numerical Experiments}

In this section, we present some numerical approximations to the blow-up time of the problem (1.1)-(1.3). We approximate the solution $u$ of the problem (1.1)-(1.3) by the solution $U_{h}^{(n)}$ of the following explicit scheme:

$$
\begin{gathered}
\frac{U_{i}^{(n+1)}-U_{i}^{(n)}}{\Delta t_{n}}=\delta^{2} U_{i}^{(n)}+f\left(U_{I}^{(n)}\right), \quad 1 \leq i \leq I, \\
U_{0}^{(n)}=0, \\
U_{i}^{(0)}=\varphi_{i} \geq 0, \quad 0 \leq i \leq I,
\end{gathered}
$$

where

$$
\Delta t_{n}=\min \left\{\frac{h^{2}}{3} ; \frac{\tau}{f\left(\left\|U_{h}^{(n)}\right\|_{\infty}\right)}\right\} .
$$


We can also approximate the solution $u$ of the problem (1.1)-(1.3) by the solution $U_{h}^{(n)}$ of the following implicit scheme:

$$
\begin{gathered}
\frac{U_{i}^{(n+1)}-U_{i}^{(n)}}{\Delta t_{n}}=\delta^{2} U_{i}^{(n+1)}+f\left(U_{I}^{(n)}\right), \quad 1 \leq i \leq I, \\
U_{0}^{(n)}=0, \\
U_{i}^{(0)}=\varphi_{i} \geq 0, \quad 0 \leq i \leq I,
\end{gathered}
$$

where

$$
\Delta t_{n}=\frac{\tau}{f\left(\left\|U_{h}^{(n)}\right\|_{\infty}\right)}
$$

$\tau=h^{2}$. In both cases, we take $\varphi_{i}=\varepsilon \sin \left(\frac{i \pi h}{2}\right), 1 \leq i \leq I$. The explicit scheme may be written as follows:

$$
\begin{gathered}
U_{i}^{(n+1)}=U_{i}^{(n)}+\Delta t_{n} \delta^{2} U_{i}^{(n+1)}+\Delta t_{n} f\left(U_{i}^{(n)}\right), \\
U_{i}^{(n+1)}=U_{i}^{(n)}+\Delta t_{n} \frac{U_{i+1}^{(n)}-2 U_{i}^{(n)}+U_{i-1}^{(n)}}{h^{2}}+\Delta t_{n} f\left(U_{i}^{(n)}\right), \\
U_{i}^{(n+1)}=\left(1-\frac{2 \Delta t_{n}}{h^{2}}\right) U_{i}^{(n)}+\frac{\Delta t_{n}}{h^{2}}\left(U_{i+1}^{(n)}+U_{i-1}^{(n)}\right)+\Delta t_{n} f\left(U_{i}^{(n)}\right), \\
U_{i}^{(n+1)}=\frac{\Delta t_{n}}{h^{2}}\left(U_{i+1}^{(n)}\right)+\left(1-\frac{2 \Delta t_{n}}{h^{2}}\right) U_{i}^{(n)}+\frac{\Delta t_{n}}{h^{2}}\left(U_{i-1}^{(n)}\right)+\Delta t_{n} f\left(U_{i}^{(n)}\right) .
\end{gathered}
$$

For $i=1$,

$$
U_{1}^{(n+1)}=\frac{\Delta t_{n}}{h^{2}}\left(U_{2}^{(n)}\right)+\left(1-\frac{2 \Delta t_{n}}{h^{2}}\right) U_{1}^{(n)}+\frac{\Delta t_{n}}{h^{2}}\left(U_{0}^{(n)}\right)+\Delta t_{n} f\left(U_{1}^{(n)}\right) .
$$

For $i=1$,

$$
U_{1}^{(n+1)}=\left(1-\frac{2 \Delta t_{n}}{h^{2}}\right) U_{1}^{(n)}+\frac{\Delta t_{n}}{h^{2}}\left(U_{2}^{(n)}\right)+\Delta t_{n} f\left(U_{1}^{(n)}\right) .
$$


For $i=2$,

$$
U_{2}^{(n+1)}=\frac{\Delta t_{n}}{h^{2}}\left(U_{3}^{(n)}\right)+\left(1-\frac{2 \Delta t_{n}}{h^{2}}\right) U_{2}^{(n)}+\frac{\Delta t_{n}}{h^{2}}\left(U_{1}^{(n)}\right)+\Delta t_{n} f\left(U_{2}^{(n)}\right) .
$$

Let us notice that the restriction on the time step $\Delta t_{n} \leq \frac{h^{2}}{3}$ ensure the nonnegativity of the discrete solution.

According to the implicit scheme, it may be written in the following form:

$$
\begin{gathered}
\frac{U_{i}^{(n+1)}-U_{i}^{(n)}}{\Delta t_{n}}=\frac{U_{i+1}^{(n+1)}-2 U_{i}^{(n+1)}+U_{i-1}^{(n+1)}}{h^{2}}+f\left(U_{i}^{(n)}\right), \\
U_{0}^{(n+1)}=0 ; \quad U_{I}^{(n+1)}=0, \\
U_{i}^{(n+1)}=\frac{\Delta t_{n}}{h^{2}} U_{i}^{(n+1)}-\frac{2 \Delta t_{n}}{h^{2}} U_{i}^{(n+1)}+\frac{\Delta t_{n}}{h^{2}} U_{i}^{(n+1)}+U_{i}^{(n)}+\Delta t_{n} f\left(U_{i}^{(n)}\right) \\
\left(1+\frac{2 \Delta t_{n}}{h^{2}}\right) U_{i}^{(n+1)}-\frac{\Delta t_{n}}{h^{2}} U_{i+1}^{(n+1)}-\frac{\Delta t_{n}}{h^{2}} U_{i-1}^{(n+1)}=U_{i}^{(n)}+\Delta t_{n} f\left(U_{i}^{(n)}\right),
\end{gathered}
$$

or

$$
-\frac{\Delta t_{n}}{h^{2}} U_{i-1}^{(n+1)}+\left(1+\frac{2 \Delta t_{n}}{h^{2}}\right) U_{i}^{(n+1)}-\frac{\Delta t_{n}}{h^{2}} U_{i+1}^{(n+1)}=U_{i}^{(n)}+\Delta t_{n} f\left(U_{i}^{(n)}\right) .
$$

For $i=1$,

$$
\left(1+\frac{2 \Delta t_{n}}{h^{2}}\right) U_{1}^{(n+1)}-\frac{\Delta t_{n}}{h^{2}} U_{2}^{(n+1)}=U_{1}^{(n)}+\Delta t_{n} f\left(U_{1}^{(n)}\right) .
$$

For $i=2$,

$$
-\frac{\Delta t_{n}}{h^{2}} U_{1}^{(n+1)}+\left(1+\frac{2 \Delta t_{n}}{h^{2}}\right) U_{2}^{(n+1)}-\frac{\Delta t_{n}}{h^{2}} U_{3}^{(n+1)}=U_{2}^{(n)}+\Delta t_{n} f\left(U_{2}^{(n)}\right) .
$$


For $i=I-1$,

$$
-\frac{\Delta t_{n}}{h^{2}} U_{2}^{(n+1)}+\left(1+\frac{2 \Delta t_{n}}{h^{2}}\right) U_{I-1}^{(n+1)}=U_{1}^{(n)}+\Delta t_{n} f\left(U_{1}^{(n)}\right)
$$

lead us to the linear system below

$$
A_{h}^{(n)} U_{h}^{(n+1)}=F_{h}^{(n)}
$$

where $A_{h}^{(n)}$ is an $I \times I$ tridiagonal matrix defined as follows:

$$
A_{h}^{(n)}=\left(\begin{array}{lllll}
1+2 \frac{\Delta t_{n}}{h^{2}} & -\frac{\Delta t_{n}}{h^{2}} & 0 & \cdots & 0 \\
-\frac{\Delta t_{n}}{h^{2}} & 1+2 \frac{\Delta t_{n}}{h^{2}} & -\frac{\Delta t_{n}}{h^{2}} & 0 & \cdots \\
0 & \ddots & \ddots & \ddots & \ddots \\
\vdots & \ddots & \ddots & \ddots & -\frac{\Delta t_{n}}{h^{2}} \\
0 & \cdots & 0 & -\frac{\Delta t_{n}}{h^{2}} & 1+2 \frac{\Delta t_{n}}{h^{2}}
\end{array}\right)
$$

implies that

$$
A_{h}^{(n)}=\left(\begin{array}{ccccc}
a_{0} & b_{0} & 0 & \ldots & 0 \\
c_{0} & a_{0} & b_{0} & 0 & \ldots \\
0 & \ddots & \ddots & \ddots & \ddots \\
\vdots & \ddots & \ddots & \ddots & b_{0} \\
0 & \ldots & 0 & c_{0} & a_{0}
\end{array}\right),
$$

with

$$
\begin{gathered}
a_{0}=1+2 \frac{\Delta t_{n}}{h^{2}}, \\
b_{0}=-\frac{\Delta t_{n}}{h^{2}}, \quad i=1, \ldots, I-2, \\
c_{0}=-\frac{\Delta t_{n}}{h^{2}}, \quad i=1, \ldots, I-1,
\end{gathered}
$$


$\left(F^{(n)}\right)_{i}-U_{i}^{(n)}+\Delta t_{n} f\left(U_{i}^{(n)}\right)$ and $A_{h}^{(n)}$ a three-diagonal matrix verifying the following properties:

$\left(A_{h}^{(n)}\right)_{i, i}=\left(1+\frac{2 \Delta t_{n}}{h^{2}}\right)>0,0 \leq i \leq I$ and $\left(A_{h}^{(n)}\right)_{i-1, i}=-\frac{\Delta t_{n}}{h^{2}}=\left(A_{h}^{(n)}\right)_{i, i+1} \leq 0$,

$2 \leq i \leq I-2$ so that $\left(A_{h}^{(n)}\right)_{i, i} \geq \sum_{i \neq j}\left|\left(A_{h}^{(n)}\right)_{i, j}\right|$.

It follows that $U_{h}^{(n)}$ exists for $n \geq 0$. In addition, since $U_{h}^{(0)}$ is nonnegative, $U_{h}^{(n)}$ is also nonnegative for $n \geq 0$.

We need the following definition.

Definition 6.1. The discrete solution $U_{h}^{(n)}$ of the explicit or of the implicit scheme blows up in a finite time if $\lim _{n \rightarrow+\infty}\left\|U_{h}^{(n)}\right\|_{\infty}=+\infty$ and the series $\sum_{n=0}^{+\infty} \Delta t_{n}$ converges. The quantity $\sum_{n=0}^{+\infty} \Delta t_{n}$ is called the numerical blow-up time of the solution $U_{h}^{(n)}$.

In the following tables, in rows, we present the numerical blow-up time, values of $n$, the CPU time and the orders of the approximations corresponding to the meshes of $16,32,64,128,256,512$.

We take for the numerical blow-up time $t_{n}=\sum_{j=0}^{n-1} \Delta t_{j}$ which is computed at the first time when

$$
\Delta t_{n}=\left|T_{n+1}-T_{n}\right| \leq 10^{-16} .
$$

The initial condition is $\varphi_{i}=\varepsilon \sin \left(\frac{i \pi h}{2}\right), 1 \leq i \leq I$. The order(s) of the method is computed from

$$
s=\frac{\log \left(\left(T_{4 h}-T_{2 h}\right) /\left(T_{2 h}-T_{h}\right)\right)}{\log (2)} .
$$


For the numerical values, we take, $U_{i}^{(0)}=\varphi_{i}=\varepsilon \sin \left(\frac{i \pi h}{2}\right)$.

Numerical experiments for $f(u)=\beta e^{U_{I}^{(n)}}$.

First case: $\beta=5 ; \varphi_{i}=\sin \left(\frac{i \pi h}{2}\right)$.

Table 1. Numerical blow-up times, numbers of iterations, CPU times (seconds) and orders of the approximations obtained with the implicit Euler method

\begin{tabular}{|l|l|l|l|l|}
\hline 16 & 0.200507 & 1688 & 2 & - \\
\hline 32 & 0.206603 & 6496 & 6 & - \\
\hline 64 & 0.211148 & 24932 & 21 & 1.50 \\
\hline 128 & 0.211205 & 33708 & 45 & 2.00 \\
\hline
\end{tabular}

Table 2. Numerical blow-up times, numbers of iterations, CPU times (seconds) and orders of the approximations obtained with the explicit Euler method

\begin{tabular}{|l|l|l|l|l|}
\hline$I$ & $t_{n}$ & $n$ & CPUt & $s$ \\
\hline 16 & 0.200514 & 1689 & 2 & - \\
\hline 32 & 0.206628 & 6502 & 6 & - \\
\hline 64 & 0.211201 & 24939 & 21 & 1.50 \\
\hline 128 & 0.211215 & 33708 & 45 & 2.00 \\
\hline
\end{tabular}

Second case: $\beta=10, \varepsilon=0$.

Table 3. Numerical blow-up times, numbers of iterations, CPU times (seconds) and orders of the approximations obtained with the implicit Euler method

\begin{tabular}{|l|l|l|l|l|}
\hline 16 & 0.111327 & 843 & 1 & - \\
\hline 32 & 0.110838 & 3228 & 5 & - \\
\hline 64 & 0.111079 & 12350 & 24 & 1.57 \\
\hline 128 & 0.111088 & 13956 & 33 & 1.58 \\
\hline
\end{tabular}


Table 4. Numerical blow-up times, numbers of iterations, CPU times (seconds) and orders of the approximations obtained with the explicit Euler method

\begin{tabular}{|l|l|l|l|l|}
\hline$I$ & $t_{n}$ & $n$ & CPUt & $s$ \\
\hline 16 & 0.111327 & 843 & 1 & - \\
\hline 32 & 0.110838 & 3228 & 8 & - \\
\hline 64 & 0.111149 & 12402 & 34 & 1.57 \\
\hline 128 & 0.111188 & 13989 & 38 & 1.58 \\
\hline
\end{tabular}

Third case: $\beta=20 ; \varepsilon=0$.

Table 5. Numerical blow-up times, numbers of iterations, CPU times (seconds) and orders of the approximations obtained with the implicit Euler method

\begin{tabular}{|l|l|l|l|l|}
\hline 16 & 0.052386 & 418 & 1 & - \\
\hline 32 & 0.051354 & 1596 & 5 & - \\
\hline 64 & 0.051378 & 6096 & 24 & 2.00 \\
\hline 128 & 0.051106 & 23249 & 120 & 1.50 \\
\hline
\end{tabular}

Table 6. Numerical blow-up times, numbers of iterations, CPU times (seconds) and orders of the approximations obtained with the explicit Euler method

\begin{tabular}{|l|l|l|l|l|}
\hline$I$ & $t_{n}$ & $n$ & CPUt & $s$ \\
\hline 16 & 0.052389 & 454 & 1 & - \\
\hline 32 & 0.051355 & 1598 & 6 & - \\
\hline 64 & 0.051377 & 6194 & 26 & 2.00 \\
\hline 128 & 0.051115 & 23255 & 124 & 1.50 \\
\hline
\end{tabular}


Fourth case: $\beta=100 ; \varepsilon=0$.

Table 7. Numerical blow-up times, numbers of iterations, CPU times (seconds) and orders of the approximations obtained with the implicit Euler method

\begin{tabular}{|l|l|l|l|l|}
\hline 16 & 0.011277 & 84 & 1 & - \\
\hline 32 & 0.010322 & 319 & 3 & - \\
\hline 64 & 0.010080 & 1216 & 23 & 1.70 \\
\hline 128 & 0.001025 & 3708 & 30 & 2.00 \\
\hline
\end{tabular}

Table 8. Numerical blow-up times, numbers of iterations, CPU times (seconds) and orders of the approximations obtained with the explicit Euler method

\begin{tabular}{|l|l|l|l|l|}
\hline$I$ & $t_{n}$ & $n$ & $\mathrm{CPUt}$ & $s$ \\
\hline 16 & 0.011277 & 84 & 1 & - \\
\hline 32 & 0.010322 & 319 & 3 & - \\
\hline 64 & 0.010080 & 1216 & 23 & 1.70 \\
\hline 128 & 0.010020 & 4633 & 30 & 2.00 \\
\hline
\end{tabular}

Remark 6.1. In the case where the initial data is null, $\phi=0$, and the reaction term increases as a function of $\beta$ it is not hard to see that the blow-up time of the solution equals $\frac{1}{\beta}$. We observe from Tables 1-6 that the numerical blow-up time tends to $\frac{1}{\beta}$ for $\beta=10, \beta=20$, and $\beta=100$.

When $\varphi_{i}=\sin \left(\frac{i \pi h}{2}\right)$ with $\beta=5$ it is not hard to see that the blowup time of the solution equals $\frac{1}{\beta}$. See the Tables 7-8.

In the following, we also give some plots to illustrate our analysis. In Figures 1 to 6 , we can appreciate that the discrete solution blows up globally. Let us notice that, theoretically, we know that the continuous solution blows up globally under the assumptions given in the introduction of the present paper (see [17], [46]) 


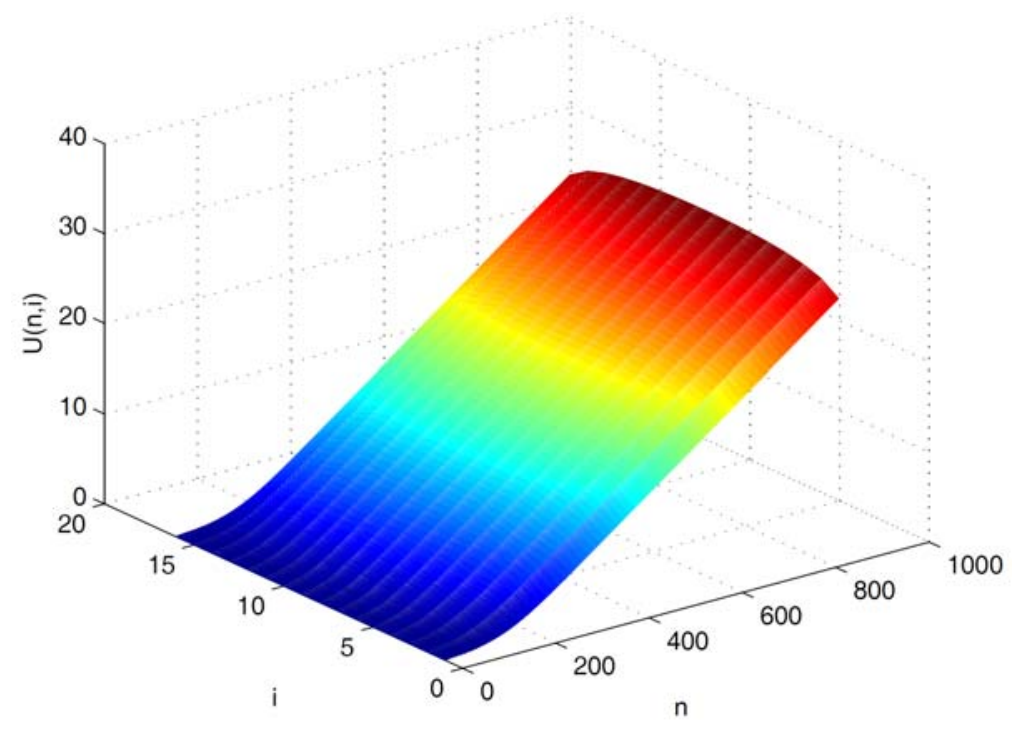

Figure 1. Evolution of the discrete solution, source $f(u)=\beta e^{u}, \beta=10$, $\varepsilon=0, I=16$ (implicit scheme).

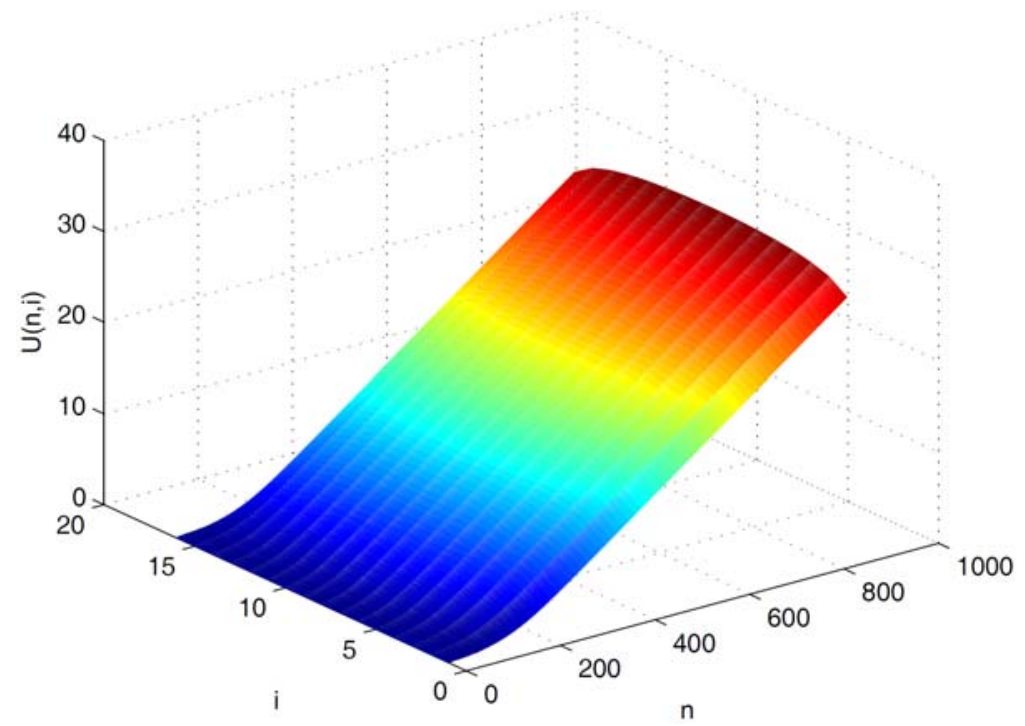

Figure 2. Evolution of the discrete solution, source $f(u)=\beta e^{u}, \beta=10$, $\varepsilon=0, I=16$ (explicit scheme). 


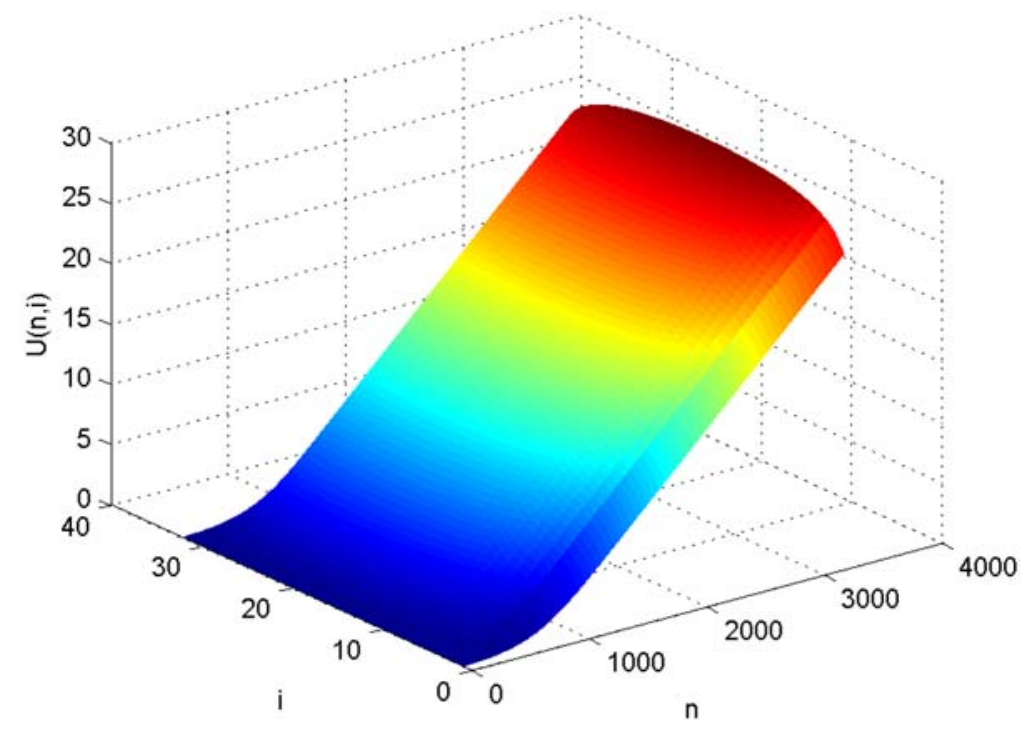

Figure 3. Evolution of the discrete solution, source $f(u)=\beta e^{u}, \beta=10$, $\varepsilon=0, I=32$ (implicit scheme).

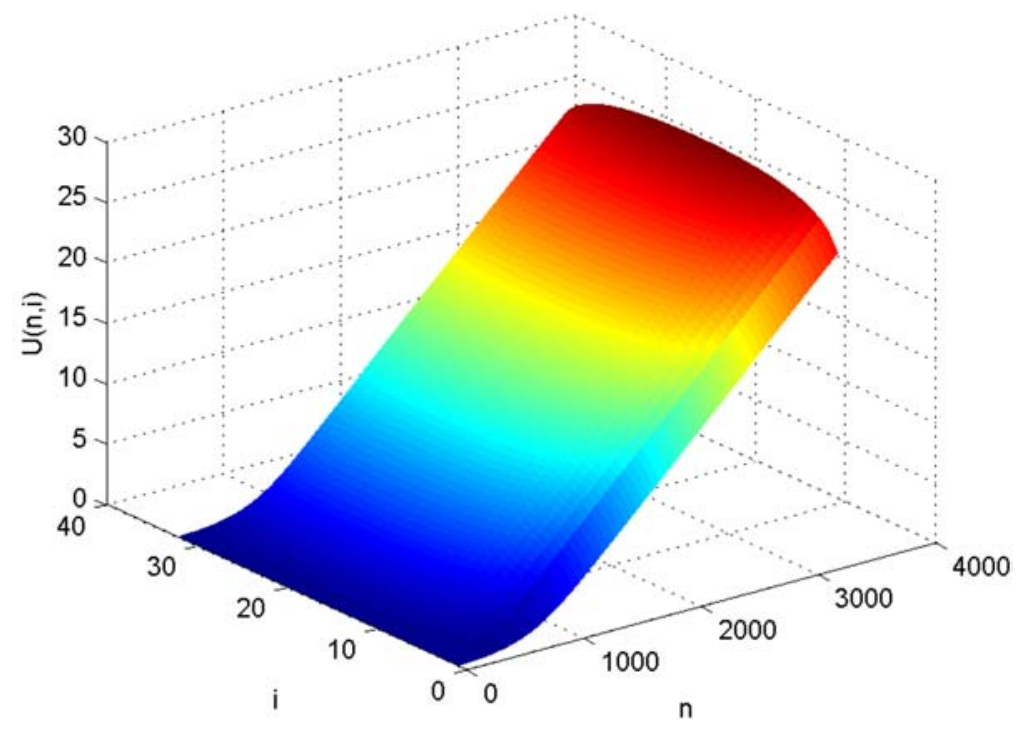

Figure 4. Evolution of the discrete solution, source $f(u)=\beta e^{u}, \beta=10$, $\varepsilon=0, I=32$ (explicit scheme). 


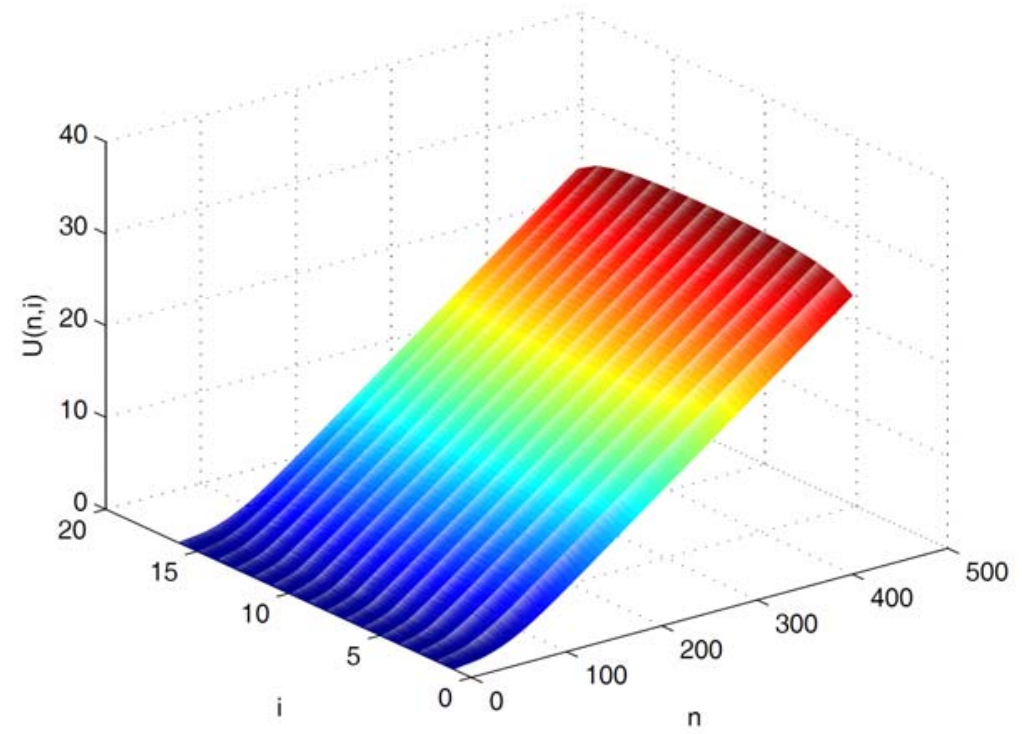

Figure 5. Evolution of the discrete solution, source $f(u)=\beta e^{u}, \beta=20, \varepsilon=0$ (implicit scheme).

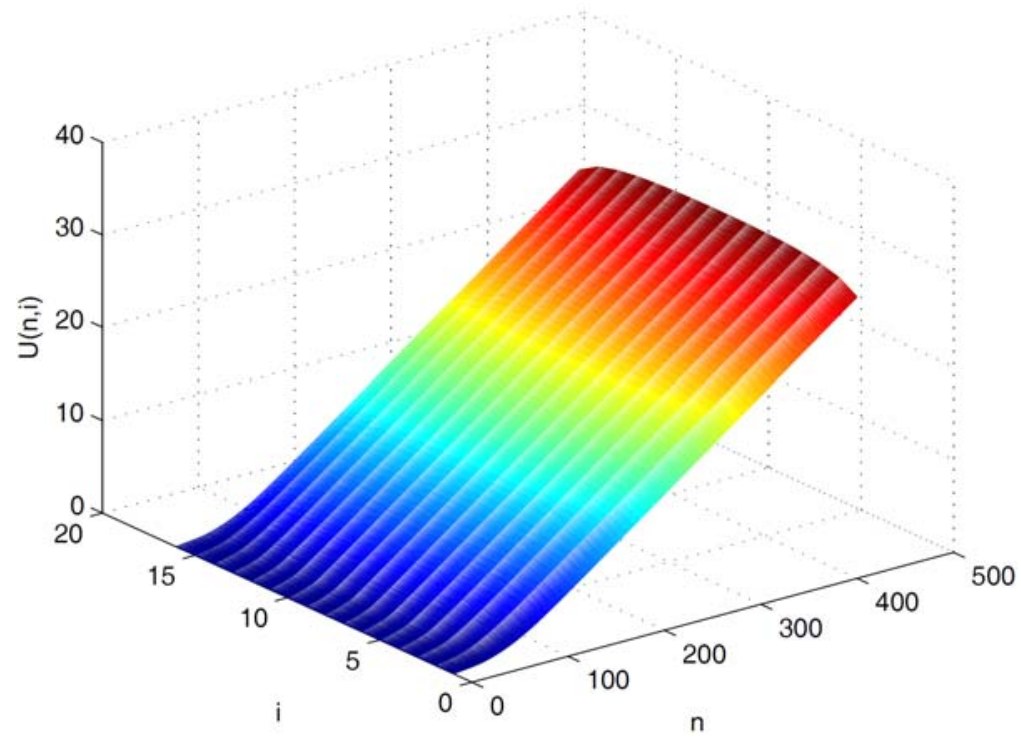

Figure 6. Evolution of the discrete solution, source $f(u)=\beta e^{u}, \beta=20, \varepsilon=0$ (explicit scheme). 


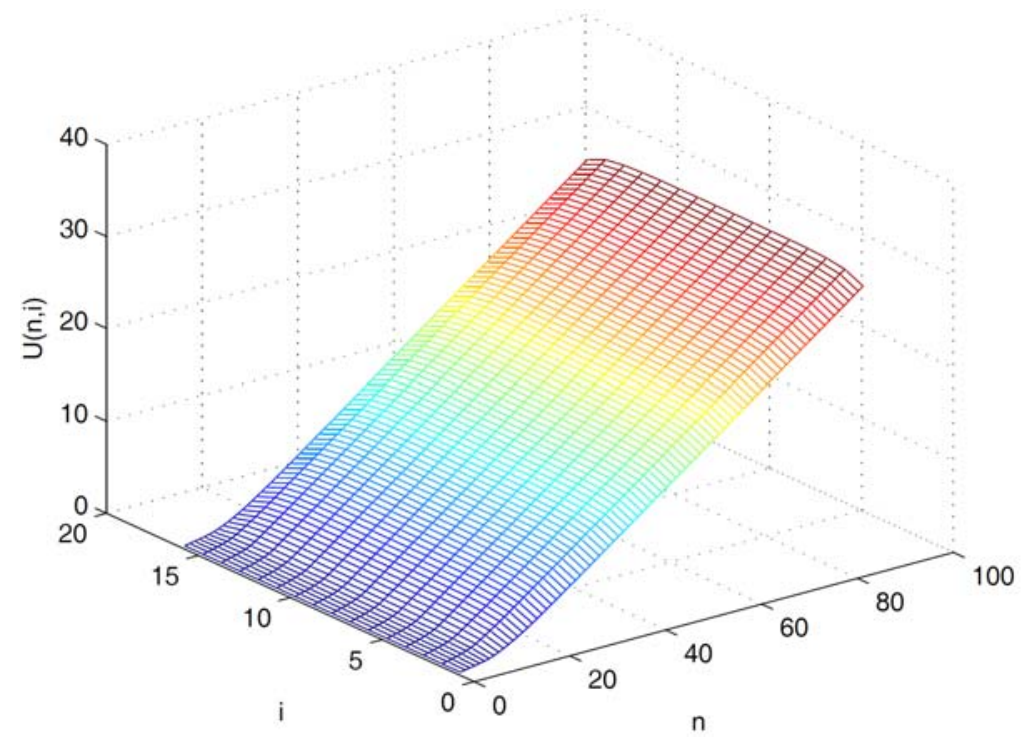

Figure 7. Evolution of the discrete solution, source $f(u)=\beta e^{u}, \beta=100$, $\varepsilon=0, I=16$ (implicit scheme).

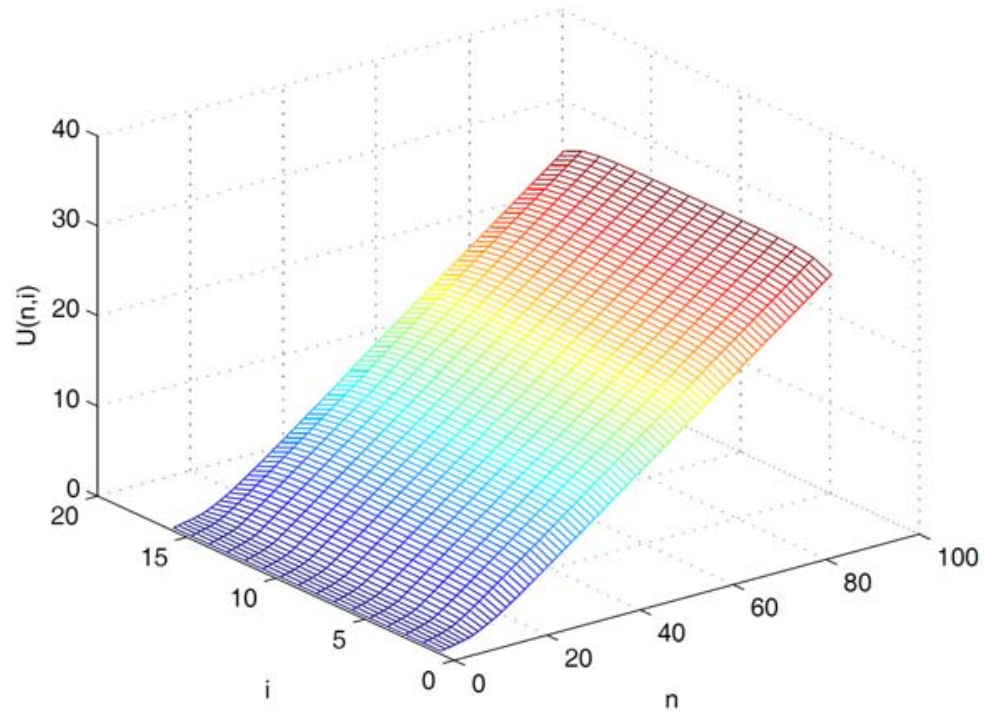

Figure 8. Evolution of the discrete solution, source $f(u)=\beta e^{u}, \beta=100$, $\varepsilon=0, I=16$ (explicit scheme). 


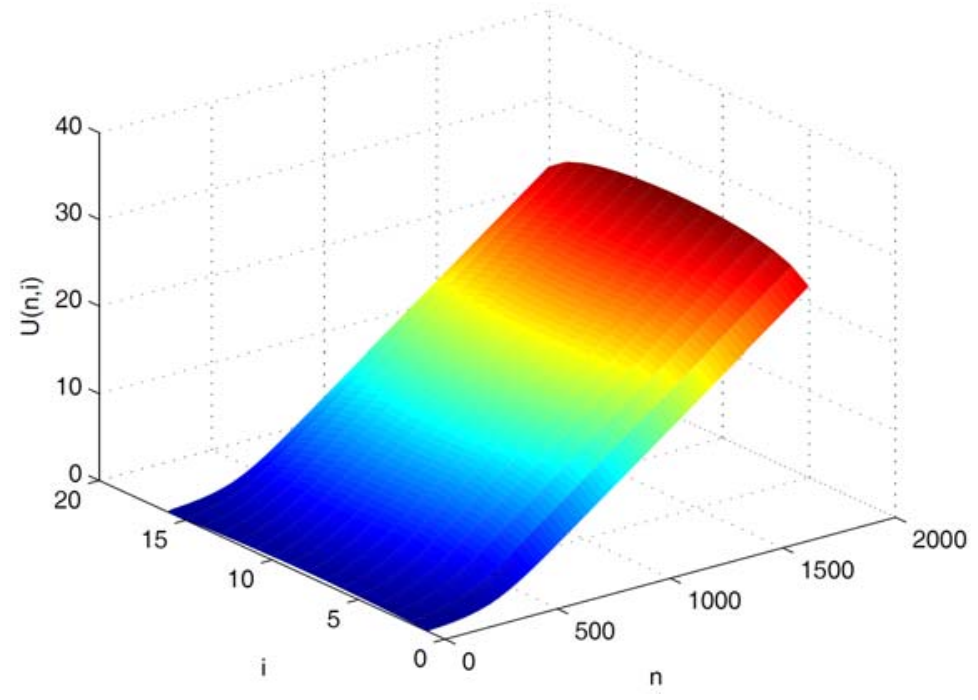

Figure 9. Evolution of the discrete solution, source $f(u)=\beta e^{u}, \beta=5$, $\varphi_{i}=\sin \left(\frac{i \pi h}{2}\right), I=16$ (implicit scheme).

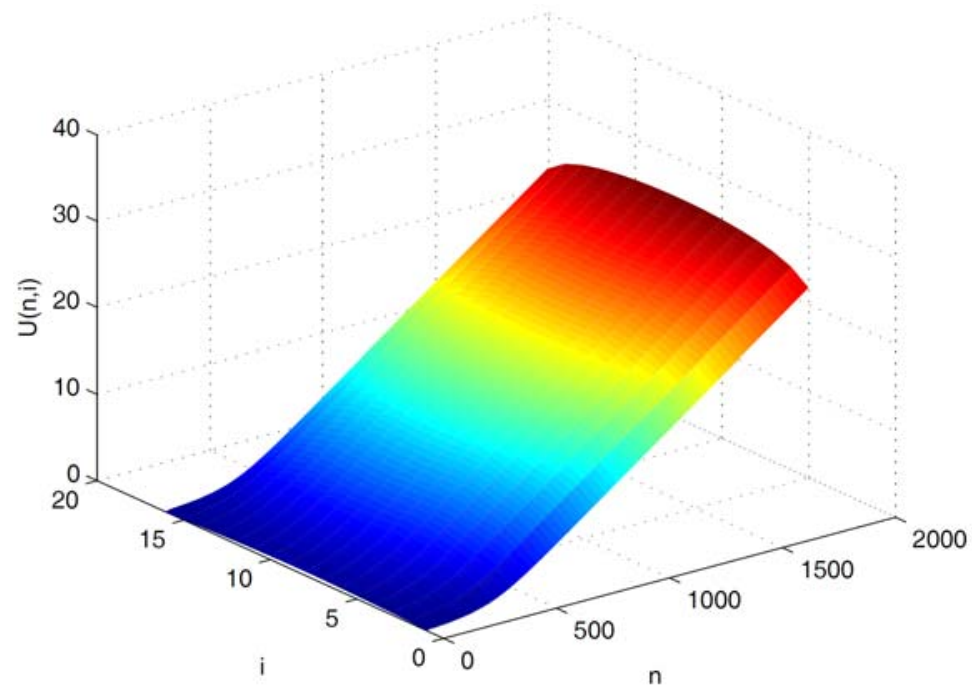

Figure 10. Evolution of the discrete solution, source $f(u)=\beta e^{u}, \beta=5$, $\varphi_{i}=\sin \left(\frac{i \pi h}{2}\right), I=16$ (explicit scheme). 


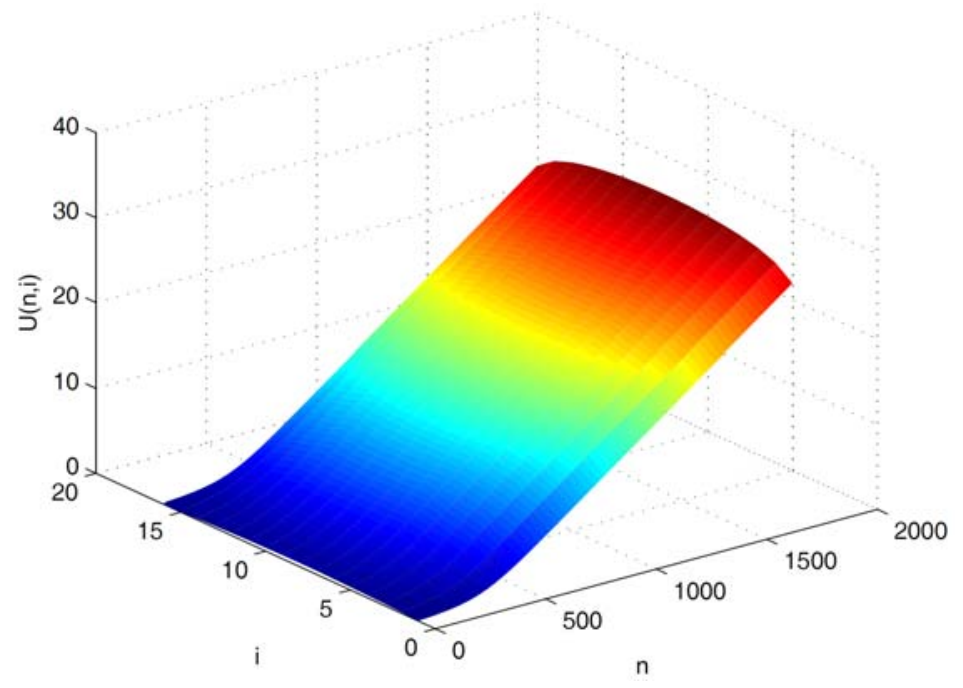

Figure 11. Evolution of the discrete solution, source $f(u)=\beta e^{u}, \beta=5$, $\varphi_{i}=\sin \left(\frac{i \pi h}{2}\right), I=32$ (implicit scheme).

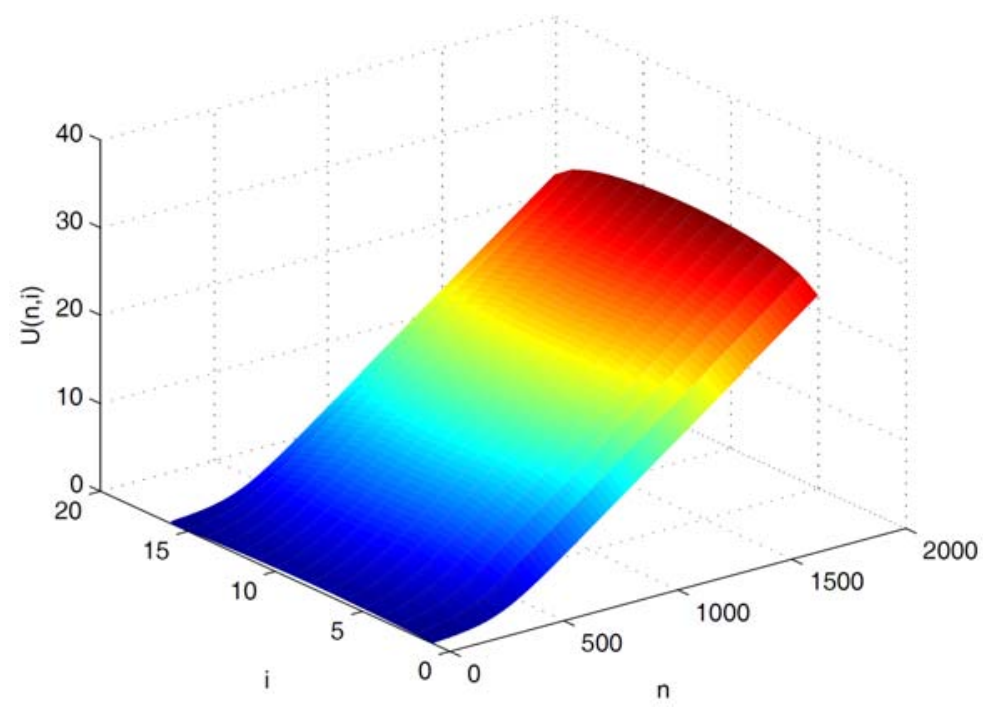

Figure 12. Evolution of the discrete solution, source $f(u)=\beta e^{u}, \beta=5$, $\varphi_{i}=\sin \left(\frac{i \pi h}{2}\right), I=32$ (explicit scheme). 


\section{Acknowledgement}

The authors want to thank the anonymous referee for the throughout reading of the manuscript and several suggestions that help us improve the representation of the paper.

\section{References}

[1] L. M. Abia, J. C. López-Marcos and J. Martinez, On the blow-up time convergence of semidiscretizations of reaction-diffusion equations, Applied Numerical Mathematics 26(4) (1998), 399-414.

DOI: https://doi.org/10.1016/S0168-9274(97)00105-0

[2] L. M. Abia, J. C. López-Marcos and J. Martínez, Blow-up for semidiscretizations of reaction-diffusion equations, Applied Numerical Mathematics 20(1-2) (1996), $145-156$.

DOI: https://doi.org/10.1016/0168-9274(95)00122-0

[3] G. Acosta, J. Fernandez Bonder, P. Groisman and J. D. Rossi, Simultaneous vs. nonsimultaneous blow-up in numerical approximations of a parabolic system with nonlinear boundary conditions, M2AN Mathematical Modelling and Numerical Analysis 36(1) (2002), 55-68.

DOI: https://doi.org/10.1051/m2an:2002003

[4] G. Acosta, J. Fernandez Bonder, P. Groisman and J. D. Rossi, Numerical approximation of a parabolic problem with a nonlinear boundary condition in several space dimensions, Discrete \& Continuous Dynamical Systems: B (2)2 (2002), 279-294.

DOI: https://doi.org/10.3934/dcdsb.2002.2.279

[5] G. Acosta, R. G. Duran and J. D. Rossi, An adaptive time step procedure for a parabolic problem with blow-up, Computing 68(4) (2002), 343-373.

DOI: https://doi.org/10.1007/s00607-002-1449-x

[6] C. Bandle and H. Brunner, Blow-up in diffusion equations: A survey, Journal of Computational and Applied Mathematics 97(1-2) (1998), 3-22.

DOI: https://doi.org/10.1016/S0377-0427(98)00100-9

[7] M. Berger and R. V. Kohn, A rescaling algorithm for the numerical calculation of blowing up solutions, Communications on Pure and Applied Mathematics 41(6) (1988), 841-863.

DOI: https://doi.org/10.1002/cpa.3160410606 
[8] K. Bimpong-Beta, P. Ortoleva and J. Ross, Far-form-equilibrium phenomenon at local sites of reactions, Journal of Chemical Physics 60(8) (1974), 3124-3133.

DOI: https://doi.org/10.1063/1.1681498

[9] T. K. Boni, On the blow-up and the asymptotic behaviour of the solution of one semilinear parabolic equation of second order, Comptes Rendus de l'Académie des Sciences, Series I: Mathematics 326(3) (1998), 317-322.

DOI: https://doi.org/10.1016/S0764-4442(97)82987-4

[10] T. K. Boni, Extinction for discretizations of some semilinear parabolic equations, Comptes Rendus de l'Académie des Sciences, Series I: Mathematics 333(8) (2001), 795-800.

DOI: https://doi.org/10.1016/S0764-4442(01)02078-X

[11] T. K. Boni, On blow-up and asymptotic behavior of solutions to a nonlinear parabolic equation of second order with nonlinear boundary conditions, Commentationes Mathematicae Universitatis Carolinae 40(3) (1999), 457-475.

[12] C. Brandle, P. Groisman and J. D. Rossi, Fully discrete adaptive methods for a blowup problem, Mathematical Models and Methods in Applied Sciences 14(10) (2004), 1425-1450.

DOI: https://doi.org/10.1142/S0218202504003751

[13] C. Brandle, F. Quiros and J. D. Rossi, An adaptive numerical method to handle blowup in a parabolic system, Numerische Mathematik 102(1) (2005), 39-59.

DOI: https://doi.org/10.1007/s00211-005-0638-x

[14] H. Brezis, Analyse Fonctionnelle, Théorie et Application, Collection Mathematiques Appliquées Pour la Maitrise, Masson, Paris.

[15] H. Brezis, T. Cazenave, Y. Martel and A. Ramiandrisoa, Blow up for $u_{t}=u_{x x}+g(u)$ revisited, Advances in Differential Equations 1(1) (1996), 73-90.

[16] C. J. Budd, W. Huang and R. D. Russel, Moving mesh methods for problems with blow-up, SIAM Journal on Scientific Computing 17(2) (1996), 305-327.

DOI: https://doi.org/10.1137/S1064827594272025

[17] J. M. Chadam and H. M. Yin, A diffusion equation with localized chemical reactions, Proceedings of the Edinburgh Mathematical Society 37(1) (1994), 101-118.

DOI: https://doi.org/10.1017/S0013091500018721

[18] Y. G. Chen, Asymptotic behaviours of blowing up solutions for finite difference analogue of $u_{t}=u_{x x}+u^{1+\alpha}$, J. Fac. Sci. Univ. Tokyo, Sec. IA, Math. 33 (1986), $541-574$.

[19] A. De. Pablo, M. P. LLanos and R. Ferreira, Numerical blow-up for $p$-Laplacian equation with a nonlinear source, Proceedings of Equatdiff. 11 (2005), 363-367. 
[20] R. G. Duran, J. I. Etcheverry and J. D. Rossi, Numerical approximation of a parabolic problem with a nonlinear boundary condition, Discrete \& Continuous Dynamical Systems: A 4(3) (1998), 497-506.

DOI: https://doi.org/10.3934/dcds.1998.4.497

[21] C. M. Elliot and A. M. Stuart, The global dynamics of discrete semilinear parabolic equations, SIAM Journal on Numerical Analysis 30(6) (1993), 1622-1663.

DOI: https://doi.org/10.1137/0730084

[22] J. Fernandez Bonder and J. D. Rossi, Blow-up vs. spurious steady solutions, Proceedings of the American Mathematical Society 129(1) (2001), 139-144.

DOI: https://doi.org/10.1090/S0002-9939-00-05663-X

[23] J. Fernandez Bonder, P. Groisman and J. D. Rossi, On numerical blow-up sets, Proceedings of the American Mathematical Society 130(7) (2002), 2049-2055.

DOI: https://doi.org/10.1090/S0002-9939-02-06350-5

[24] R. Ferreira, P. Groisman and J. D. Rossi, Numerical blow-up for the porous medium equation with a source, Numerical Methods for Partial Differential Equations 20(4) (2004), 552-575.

\section{DOI: https://doi.org/10.1002/num.10103}

[25] V. A. Galaktionov and J. L. Vazquez, The problem of blow-up in nonlinear parabolic equations, Discrete \& Continuous Dynamical Systems: A 8(2) (2002), 399-433.

DOI: https://doi.org/10.3934/dcds.2002.8.399

[26] P. Groisman, Totally discrete explicit and semi-implicit Euler methods for a blow-up problem in several space dimensions, Computing 76(3-4) (2006), 325-352.

DOI: https://doi.org/10.1007/s00607-005-0136-0

[27] P. Groisman and J. D. Rossi, Dependance of the blow-up time with respect to parameters and numerical approximations for a parabolic problem, Asymptotic Analysis 37(1) (2004), 79-91.

[28] P. Groisman and J. D. Rossi, Asymptotic behaviour for a numerical approximation of a parabolic problem with blowing up solutions, Journal of Computational and Applied Mathematics 135(1) (2001), 135-155.

DOI: https://doi.org/10.1016/S0377-0427(00)00571-9

[29] O. A. Ladyzenskaja, V. A. Solonnikov and N. N. Ural'ceva, Linear and Quasi-Linear Equations of Parabolic Type, Translations of Mathematical Monographs, 23 AMS, Providence, RI, 1968.

[30] M. N. Le Roux, Semidiscretization in time of nonlinear parabolic equations with blow-up of the solution, SIAM Journal on Numerical Analysis 131(1) (1994), 170-195.

DOI: https://doi.org/10.1137/0731009 
[31] M. N. Le Roux, Semidiscretization in time of a fast diffusion equation, Journal of Mathematical Analysis and Applications 137(2) (1989), 354-370.

DOI: https://doi.org/10.1016/0022-247X(89)90251-5

[32] T. Imai and K. Mochizuki, On blow-up of solutions for quasilinear degeneate parabolic equations, Publications of the Research Institute for Mathematical Sciences 27(5) (1991), 695-709.

DOI: https://doi.org/10.2977/prims/1195169267

[33] T. Nakagawa, Blowing up on the finite difference solution to $u_{t}=u_{x x}+u^{2}$, Applied Mathematics and Optimization 2(4) (1975), 337-350.

DOI: https://doi.org/10.1007/BF01448176

[34] T. K. Boni and Halima Nachid, Blow-up for semidiscretizations of some semilinear parabolic equations with nonlinear boundary conditions, Rev. Ivoir. Sci. Tech. 11 (2008), 61-70.

[35] T. K. Boni, Halima Nachid and Nabongo Diabate, Blow-up for discretization of a localized semilinear heat equation, Analele Stiintifice Ale Univertatii 2 (2010).

[36] Halima Nachid, Quenching for semi discretizations of a semilinear heat equation with potentiel and general non linearities, Revue d'Analyse Numerique et de Theorie de l'Approximation 2 (2011), 164-181.

[37] Halima Nachid, Full discretizations of solution for a semilinear heat equation with Neumann boundary condition, Research and Communications in Mathematics and Mathematical Sciences 1(1) (2012), 53-85.

[38] Halima Nachid, Behavior of the numerical quenching time with a potential and general nonlinearities, Journal of Mathematical Sciences: Advances and Application 15(2) (2012), 81-105.

[39] M. H. Protter and H. F. Weinberger, Maximum Principles in Differential Equations, Prentice Hall, Englewood Cliffs, NJ, 1967.

[40] P. Quittner and P. Souplet, Superlinear Parabolic Problems: Blow-up, Global Existence and Steady States, Series: Birkhauser Advanced Tests/ Basler Lehrbcher, 2007.

[41] A. M. Stuart and M. S. Floater, On the computation of blow-up, European Journal of Applied Mathematics 1(1) (1990), 47-71.

DOI: https://doi.org/10.1017/S095679250000005X

[42] A. A. Samarski, V. A. Galaktionov, S. P. Kurdyumov and A. P. Mikhailov, Blow-up in Quasilinear Parabolic Equations, Walter de Gruyter, Berlin, 1995.

[43] R. Suzuki, On blow-up sets and asymptotic behavior of interface of one dimensional quasilinear degenerate parabolic equation, Publications of the Research Institute for Mathematical Sciences 27 (1991), 375-398. 
[44] P. Souplet, Uniform blow-up profiles and boundary behavior for diffusion equations with nonlocal nonlinear source, Journal of Differential Equations 153(2) (1999), 374-406.

DOI: https://doi.org/10.1006/jdeq.1998.3535

[45] P. Souplet, Blow-up in nonlocal reaction-diffusion equations, SIAM Journal on Mathematical Analysis 29(6) (1998), 1301-1334.

DOI: https://doi.org/10.1137/S0036141097318900

[46] L. Wang and Q. Chen, The asymptotic behavior of blow-up solution of localized nonlinear equation, Journal of Mathematical Analysis and Applications 200(2) (1996), 315-321.

DOI: https://doi.org/10.1006/jmaa.1996.0207

[47] W. Walter, Differential-und Integral-ungleichungen, Springer, Berlin, 1964.

[48] F. B. Weissler, An $L^{\infty}$ blow-up estimate for a nonlinear heat equation, Communications on Pure and Applied Mathematics 38(3) (1985), 291-295.

DOI: https://doi.org/10.1002/cpa.3160380303 